\title{
On-Demand Release of Secondary Amine Bases for the Activation of Catalysts and Crosslinkers
}

\author{
Benjamin Spitzbarth, ${ }^{a}$ Rienk Eelkema ${ }^{a *}$ \\ a Department of Chemical Engineering, Delft University of Technology, Van der Maasweg 9, 2629 HZ Delft, The Netherlands.
}

We demonstrate that the species present in the equilibrium of $D C v$ ureas can be employed in reaction cascades and as triggered organocatalysts. Easily controllable stimuli like heat or addition of water shift the equilibrium towards isocyanate and free base which can function as an in situ released reagent, both catalytically and in an equimolar fashion in different reactions. While applying heat to the system leads to a reversible liberation of amine base, addition of water makes this release irreversible. We demonstrate this application of DCv ureas with two examples. Firstly, we use the liberated base to activate a protected organocatalyst for acylhydrazone formation. Secondly, this base can be employed to trigger the release of nitrile-N-oxides from chlorooximes, which can react with 4-arm PEG-thiols to form a thiohydroximate polymer gel. These findings show the utility of DCv hindered ureas beyond their application in self-healing. We envisage their use for the design of responsive soft materials that can lead to triggered reaction cascades on demand.

\section{Introduction}

This paper describes the reversible and irreversible on-demand release of secondary amine bases to activate catalysts or crosslinkers leading to reaction cascades triggered by heat or the presence of water. To demonstrate this concept, we applied dynamic covalent (DCv) urea functionalities. These moieties have been shown by the group of J. Cheng to be a versatile motif in the development of reversible and self-healing poly(ureaurethanes). ${ }^{1}$ Dynamic motifs such as DCv ureas have been extensively used for a wide range of applications in materials, imparting polymer networks with desirable properties like selfhealing. ${ }^{2,3}$ Our work shows that beyond their traditional applications, DCv moieties can also be used as dormant reagents, enabling the use of the functional groups present in the DCv equilibrium upon application of a trigger (Figure 1 ). The urea moiety has recently gained widespread attention in the field of Dynamic Covalent Chemistry (DCVC). Amides are generally considered to be very stable and are frequently employed as protecting groups in organic synthesis due to their inertness under a wide range of conditions. ${ }^{4}$ However, substituting the nitrogen in amides with increasingly bulky substituents weakens the $\mathrm{C}-\mathrm{N}$ bond, proposedly due to torsion and therefore weakening of conjugation. ${ }^{5}$ The group of J. Cheng has applied this concept of weakened amide bonds to urea units which are in equilibrium with their corresponding isocyanate-

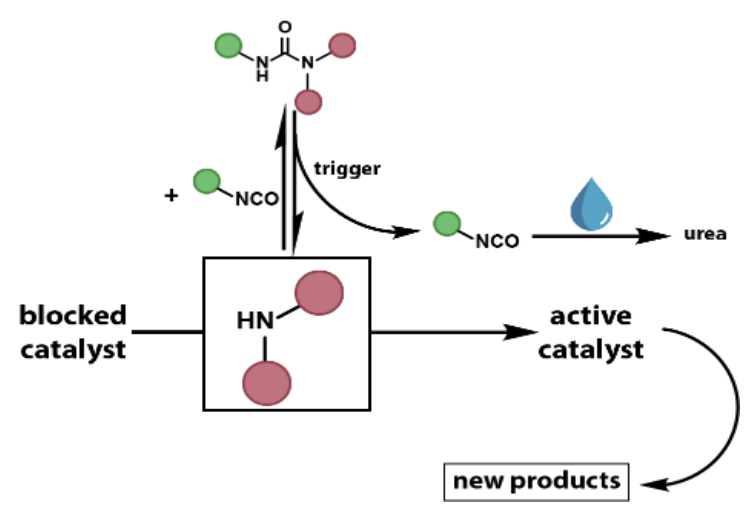

Figure 1: the base present in the equilibrium of DCv ureas can be used to deprotect a blocked organocatalyst. This can happen reversibly under application of heat or irreversibly in the presence of water. and base-building blocks to develop self-healing polymers based on the dynamic nature of this moiety. ${ }^{1}$

The concept of reversible amide, urea and urethane bonds has been widely employed in the form of 'blocked isocyanates' as a procedure to access hyperbranched polyurethanes, ${ }^{6,7}$ as well as routes to post-polymerization modifications. ${ }^{8,9}$ However, the concept of utilising the amine moiety of these 'blocked isocyanates' in the form of bulky, reversible urea functionalities opens up possibilities to design new materials responding to heat and water as stimuli.

The activation of dormant reagents has previously found some application, for instance in mechanocatalytic polymerization ${ }^{10}$, or the time-controlled $\mathrm{pH}$ lowering through the hydrolysis of glucono- $\delta$-lactone. ${ }^{11}$ Inspired by these works, the findings in this paper focus on the reversible as well as irreversible release of the secondary, bulky amine base present in dynamic urea equilibria and demonstration of its application in two different reaction cascades. In addition, we show that under the application of heat as a stimulus, the secondary amine can be used as a transient, catalytic species and return to its original, dormant urea state upon removal of the stimulus. This concept of using species which are present in DCv quilibria to trigger a reaction cascade opens up new possibilities of making use of an on demand change in chemical reactivity upon a change in conditions.

The ability to trigger the temporary or permanent release of a secondary amine base on demand enables us to make use of the basic and nucleophilic nature of these species. For example, secondary amine bases like piperidine are routinely used to remove protecting groups like the base-labile fluorenyl-methyloxycarbonyl (Fmoc) group. ${ }^{12}$ This group can be used to block catalytically active species. Triggering the DCv equilibrium of a bulky urea to release a bulky base can then lead to the 
A)

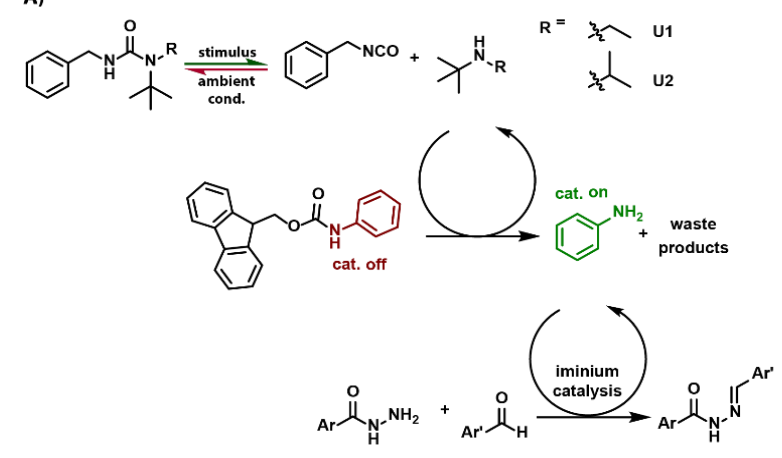

B)

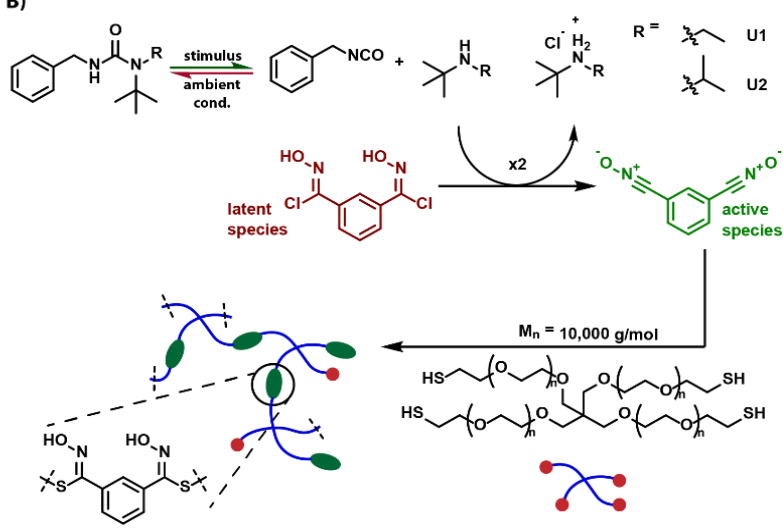

Scheme 1: two studied reaction cascades in which A) liberated base from DCV ureas $\mathbf{U} \mathbf{1}$ and $\mathbf{U} \mathbf{2}$ is used for the in situ release of aniline which in turn catalyses acylhydrazone formation and B) liberated base is used for the in situ generation of nitrile-N-oxides which leads to the formation of a thiohydroximate gel with multifunctional thiols present in solution.

deprotection of a blocked catalyst. Potential candidates for reversible blockage by the Fmoc group include nitrogen-bearing catalysts like amines. Compounds such as anilines and pyrrolidine derivatives like proline or indolines, to name a few, have been extensively studied for their catalytic function in iminium- and enamine-catalysed reactions. ${ }^{13-18}$

Another reaction in which the basicity of the liberated secondary amine bases can be applied is the elimination of hydrogen chloride from chlorooximes to form highly reactive nitrile- $N$-oxides in situ. ${ }^{19}$ This path is commonly applied to generate nitrile- $N$-oxides and has found widespread use, for example in the recently developed bioorthogonal isonitrile-chlorooxime ligation, ${ }^{20}$ or the synthesis of polyisoxazoles via click polymerisation. ${ }^{21}$ The reactivity of nitrile- $\mathrm{N}$-oxides towards many species like alkynes, alkenes and thiols makes them an attractive intermediate in reaction cascades for the formation of new species and materials alike. ${ }^{22,23}$ We describe their application for the first formation of a thiohydroximate polymer gel. This reaction is triggered by the in situ release of secondary amine bases, which generate nitrile- $N$-oxides that proceed to form an organogel in chloroform by reaction with a 4-arm PEG thiol.

We demonstrate, by means of triggered catalysis as well as triggered gelation (Scheme 1), the versatility of the DCv urea equilibrium as a starting point for a diverse range of reaction cascades.

\section{Results and Discussion}

\section{Choice of ureas and triggering conditions}

To examine the DCv equilibrium of bulky ureas under varying conditions, small molecule models had to be synthesised and tested. The group of J. Cheng showed that various bulky ureas, e.g. those based on benzyl isocyanate and two different bulky secondary amine bases, $N$-tert-butylethylamine and $N$-tert-butylisopropylamine, have fast equilibration kinetics (approx. $0.2 \mathrm{~h}^{-1}$, at $37^{\circ} \mathrm{C}$ ) and a low concentration of free base under ambient conditions $\left(20^{\circ} \mathrm{C}, K_{\text {eq }}<10^{-4} \mathrm{M}^{-1}\right){ }^{1,24}$ Furthermore, it was shown that the equilibrium reaction between isocyanate and amine base shows a temperature dependence with Arrhenius-like behaviour, ${ }^{1}$ thus making it attractive for a temperature-triggered reversible base release system. The low concentration of free base under ambient conditions is essential to avoid initiating the reaction cascades without application of the trigger. A way to irreversibly trigger the release of base is by adding intercepting agents like water or alcohols which react with the generated isocyanate and hence drive the equilibrium towards free base. These two triggers of applying heat and adding water can be combined to tune the time scale and reversibility of the base release.

\section{Triggered Fmoc deprotection for catalyst release}

To study the catalyst release, a base labile protecting group had to be employed. The ideal candidate has to be cleavable with the free base to release the cargo when a trigger is applied, but remain untouched by the miniscule amount of free base present in the DCv urea equilibrium under ambient conditions. A promising functionality for this application is the widely employed Fmoc protecting group. ${ }^{12}$ Typically, this group is cleaved by employing strongly basic conditions such as 40 vol\% piperidine. We decided to test whether this group is also cleavable under milder conditions and synthesised Fmoc-protected aniline as a model compound, probing the release of aniline via ${ }^{1} \mathrm{H}-\mathrm{NMR}$. We chose aniline as a substrate due to its easily trackable chemical shift in the ${ }^{1} \mathrm{H}-\mathrm{NMR}$ spectra, as well as its low basicity and nucleophilicity. The low basicity avoids the Fmoc-deprotection becoming self-catalysed and allows us to explicitly study the effect that the free base in the DCv urea equilibrium has without the interference of a potential second base, released in the deprotection step. As potential solvent systems, $\mathrm{CDCl}_{3}, \mathrm{CDCl}_{3} / \mathrm{MeOD}$ $(4: 1)$, DMSO- $d_{6}$ and DMSO- $d_{6} / \mathrm{D}_{2} \mathrm{O}(4: 1)$ were tested. The solutions of Fmoc-aniline and the ethyl-based DCv urea 
U1 were heated to $40{ }^{\circ} \mathrm{C}$. In DMSO- $d_{6}$ and DMSO- $d_{6} / \mathrm{D}_{2} \mathrm{O}(4: 1)$, a significant release of base and subsequently aniline could be observed (Figure 2-B, C). This proves that both an increase in temperature as well as the addition of an intercepting agent can manipulate the DCv urea equilibrium sufficiently to trigger the Fmoc-deprotection. Under ambient conditions, no such release was observed on the same timescale; neither in pure DMSO- $d_{6}$, nor upon addition of $\mathrm{D}_{2} \mathrm{O}$. In $\mathrm{CDCl}_{3}$, no changes were observed, whereas in $\mathrm{CDCl}_{3} / \mathrm{MeOD}(4: 1)$, base was released irreversibly, however only a negligible amount of free aniline was observed after two weeks. This suggests that besides the presence of base, the type of solvent used plays an important role in the deprotection step, especially at low base concentrations.

The irreversible release of base in the DMSO- $d_{6} / \mathrm{D}_{2} \mathrm{O}$-system can be attributed to the hydrolysis and subsequent decarboxylation of the free isocyanate to form benzyl amine and $N, N^{\prime}$-dibenzylurea which leads to an accumulation of free base over time (Figure $2-C$ ). ${ }^{1}$ In the pure DMSO- $d_{6}$ system however, where no accumulation of free base due to hydrolysis of the free isocyanate could be observed over time, aniline was also released (Figure 2-B). This suggests that the base is reversibly released, performs the Fmoc deprotection, but remains in equilibrium with the corresponding urea via reaction with the free isocyanate. To further demonstrate that the release of aniline truly proceeds due to the presence of reversibly or irreversibly released base from the DCv urea species, we synthesised a non-bulky non-DCv urea and subjected a solution of this urea U3 with Fmoc-aniline to the same conditions (Scheme 2). Indeed, we found no release of aniline over 48 hours at $40{ }^{\circ} \mathrm{C}$, offering further proof that the DCV properties of ureas $\mathbf{U} \mathbf{1}$ and $\mathbf{U} \mathbf{2}$ are responsible for the observed release of aniline.

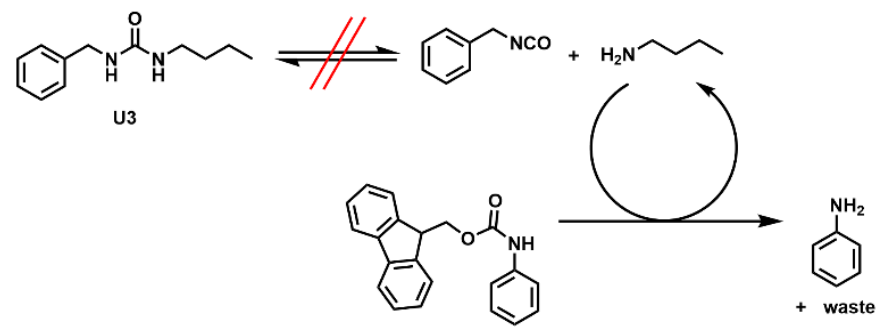

Scheme 2: non-DCv ureas with non-bulky residues do not release base under the same conditions as DCv ureas U1 and U2 do.

We hypothesise that the reason for the vastly different aniline release-kinetics in different solvent systems lies in the mechanism of the Fmoc-deprotection. As it proceeds via an E1cb elimination, a charged species is the first intermediate. This intermediate is more favoured in a polar than in an apolar medium. Hence, DMSO- $d_{6}$ and DMSO$d_{6} / D_{2} \mathrm{O}$ are suitable solvent systems for the triggered reversible and irreversible release of bulky secondary amine bases respectively. Next, we studied the influence of the substituents on the base as well as the amount of water added to the solvent system.

Table 1: rate constants for the Fmoc-deprotection of Fmoc-aniline with varying bases and solvent systems, determined via ${ }^{1} \mathrm{H}-\mathrm{NMR}$. The rate constants were retrieved by assuming pseudo first order kinetics (see S.I.). Conditions: $5 \mathrm{mM} \mathrm{Fmoc-aniline,} 50 \mathrm{mM}$ base, $40^{\circ} \mathrm{C}$.

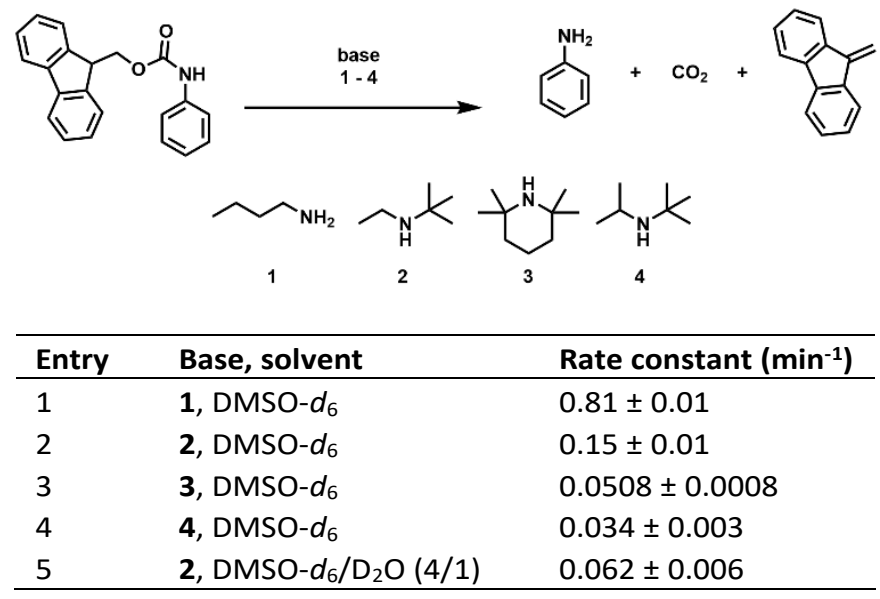

The results (Table 1 ) show that adding $\mathrm{D}_{2} \mathrm{O}$ to DMSO- $d_{6}$ decreases the reaction rate, while less bulky substituents on the amines increase the reaction rate of aniline release. Specifically, 1-butylamine in pure DMSO- $d_{6}$ leads to the fastest release, whereas $N$-tert-butylisopropylamine with its two bulky residues and rotational freedom leads to the slowest release of aniline. 
The fact that adding water slows down the release is hypothesised to be due to the fact that water is able to protonate the anionic intermediate in the E1cb elimination reaction, reforming the starting material. Thus, the cargo release is fastest in a polar solvent that does not act as a source of protons under the applied conditions.

We proceeded to study the effect that varying the bulk on the DCv ureas has on the irreversible release of base and aniline over time (Figure 2-C, D). While in experiment $C$, the base is released more slowly than in experiment $D$, the release of cargo still occurs on the same time scale. This effect is caused by two counteracting influences. Firstly, the equilibrium is shifted towards the urea more strongly in the case of less hindered urea U1. Thus, less free isocyanate is present at equilibrium which slows down the irreversible isocyanate hydrolysis, hence releasing the base over a longer time span. Secondly, the Fmoc deprotection proceeds faster with less bulky bases, thus releasing aniline faster than in the case of releasing bulkier bases in situ. These findings suggest that besides solvent composition and the presence of isocyanate intercepting agents, the bulkiness of the DCV ureas (U1 and U2) can also be used to tune the release kinetics of the cargo.

In Figure 2-C, D, it can also be seen that the release of aniline proceeds with an 'activation time', during which the rate at which aniline is released is increasing.

hen plotting the first derivative of the conversion graphs over time (see S.I.), a characteristic peak, after which the change in rate decreases, can be seen. We used this peak to define an 'activation time' for the system. This simple method of observing the effect that triggered DCv ureas have on a different reaction offers a new way to determine the dynamicity of DCv ureas relative to one another. The different values for the two DCv systems show that the more dynamic urea U2 leads to a significantly shorter activation time. Since this behaviour cannot be observed for the water-free system (Figure 2 B), we hypothesise that the reason for this activation time is the delayed accumulation of base over time. In the water-free environment, only the equilibration between DCv urea and free base, which happens on a relatively fast time scale, ${ }^{1}$ is required for the deprotection step to proceed.

In addition, we subjected the aniline release system with DCV urea U1 to a temperature ramp programme where we varied the temperature between $25^{\circ} \mathrm{C}$ and $40{ }^{\circ} \mathrm{C}$ (Figure $2 \mathrm{E}$ ). In an aqueous environment the accumulation of base is negligible under ambient conditions but leads to an irreversible accumulation at $40{ }^{\circ} \mathrm{C}$. This leads to a great degree of control over the release of precise amounts of base even through a relatively small change in temperature. Furthermore, the release of aniline is also significantly retarded initially. During the first heating cycle, the release of aniline can be observed. However, even after the first heating cycle, the release of aniline continues slowly even under ambient conditions due to the irreversible release of base, which continues to react with Fmocaniline to produce aniline.

\section{Triggered acylhydrazone formation}

The triggered aniline release system was then applied to the formation of acylhydrazones as a triggered catalysis system..$^{25-27}$ Specifically, we chose to study the formation of the acylhydrazone formed from benzhydrazide and 4nitrobenzaldehyde, which has been studied in our group and shown to proceed significantly faster in the presence of aniline. ${ }^{28}$ The conversion was monitored via ${ }^{1} \mathrm{H}-\mathrm{NMR}$ spectroscopy. Searching for a suitable solvent system to run this reaction cascade (Scheme 1-A) in, we found that using pure DMSO- $d_{6}$ resulted in negligible conversion and that adding $\mathrm{D}_{2} \mathrm{O}$ is crucial in reaching high conversions to the corresponding acylhydrazone in all experiments. We propose that this is due to the $\mathrm{H}$-bond donor capabilities of water and the importance in activating the aldehyde for nucleophilic attack. Hence, $4 / 1$ DMSO- $d_{6} / D_{2} \mathrm{O}$ was used in the experiments where acylhydrazone formation was probed via ${ }^{1} \mathrm{H}-\mathrm{NMR}$. We studied the formation in the absence of catalysts (i.e. background reaction), the direct influence of $100 \mathrm{~mol} \%$ aniline, the influence of 5 eq. DCv hindered urea in combination with 100 mol\% Fmoc-aniline, as well as the influence of 5 eq. DCv hindered urea without addition of any (pre-)catalyst. The results of these studies (Figure 3) show that the addition of aniline as a catalyst significantly speeds up the formation of acylhydrazone over the background reaction, as expected. However, even the DCV hindered urea U1 by itself speeds up the reaction and a combination of $\mathbf{U} \mathbf{1}$ with pre-catalyst Fmoc-aniline as a triggered release system leads to the largest increase in reaction rate. This surprising result suggests that another catalytically active species must be present or produced in the early stages of the release reactions. While the catalytic action of the aniline that is released from Fmocaniline is well understood, ${ }^{18}$ we decided to test whether the bulky base $\mathbf{2}$ released from DCv urea U1 as well as a simple non-dynamic urea moiety (i.e. H-bonding catalyst) can also catalyse the acylhydrazone formation. While the non-dynamic urea showed no formation above background levels (see S.I.), the free bulky base $\mathbf{2}$ showed a catalytic effect on the acylhydrazone formation. This observation explains why the reaction catalysed by a combination of Fmoc-aniline and DCV urea U1 shows a higher maximum rate than the aniline-catalysed reaction. This proves that the triggered DCv urea systems can not only be used to release catalysts but also enhance nucleophilic catalysis above rates found with the free catalyst alone. 
A)
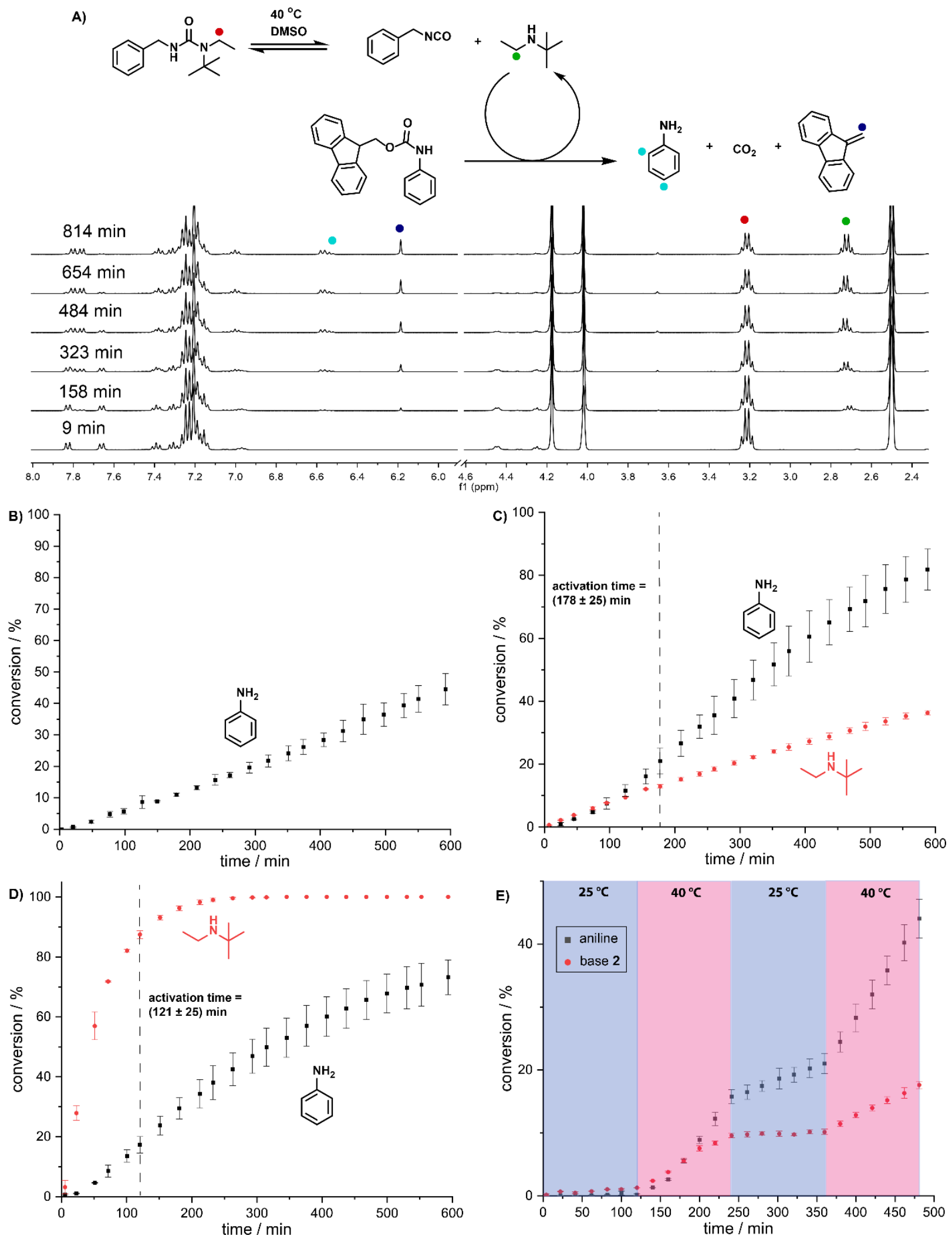

Figure 2: A) the studied reaction cascade: conversion of Fmoc-aniline and $\mathrm{DCV}$ urea, leading to release of aniline and base over time, tracked via ${ }^{1} \mathrm{H}-\mathrm{NMR}$ with application of temperature and water as triggers. B) conversion of Fmoc-aniline $(5 \mathrm{mM})$ to aniline in DMSO- $\mathrm{d}_{6}$ at $40{ }^{\circ} \mathrm{C}$ in the presence of ethyl-based $\mathrm{DCV}$ urea $\mathbf{U 1}(50 \mathrm{mM})$ as determined by NMR. No base release could be observed. C) conversion of Fmoc-aniline ( $5 \mathrm{mM}$ ) to aniline and irreversible release of $\mathrm{N}$-tert-butylethylamine from ethylbased DCv urea U1 $(50 \mathrm{mM})$ in DMSO- $\mathrm{d}_{6} / \mathrm{D}_{2} \mathrm{O}(4: 1)$ at $40^{\circ} \mathrm{C}$ as determined by NMR. D) conversion of Fmoc-aniline ( $\left.5 \mathrm{mM}\right)$ to aniline and irreversible release of $\mathrm{N}$-tertbutylisopropylamine from isopropyl-based DCv urea $\mathbf{U 2}(50 \mathrm{mM})$ in DMSO- $\mathrm{d}_{6} / \mathrm{D}_{2} \mathrm{O}(4: 1)$ at $40{ }^{\circ} \mathrm{C}$ as determined via NMR. The dashed line marks the activation time of the $\mathrm{DCV}$ systems in C) and D) (see S.I.). E) conversion of Fmoc-aniline (5 mM) to aniline and irreversible release of $\mathrm{N}$-tert-butylethylamine from ethyl-based DCv urea U1 $(50 \mathrm{mM})$ in DMSO- $\mathrm{d}_{6} / \mathrm{D}_{2} \mathrm{O}(4: 1)$ at varying temperature over time as determined by NMR. 


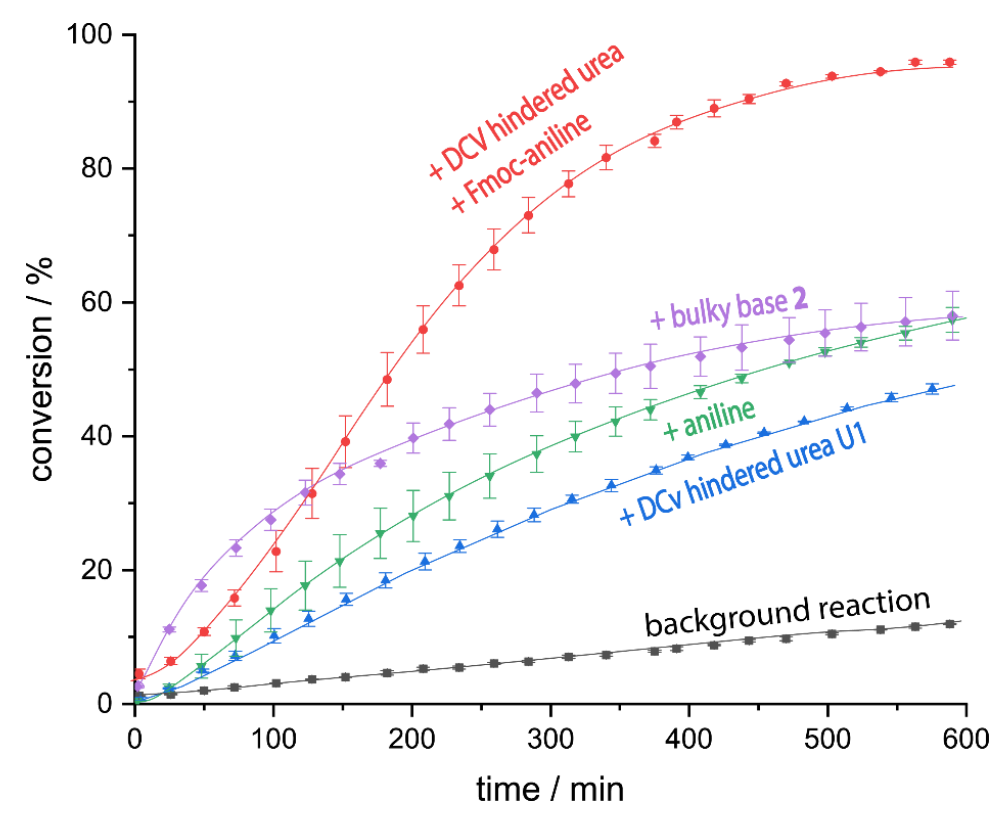

Figure 3: formation of acylhydrazone from benzhydrazide and 4-nitrobenzaldehyde over time in 4:1 DMSO- $\mathrm{d}_{6} / \mathrm{D}_{2} \mathrm{O}$ at $40{ }^{\circ} \mathrm{C}$. Black squares: background reaction between benzhydrazide $(10 \mathrm{mM})$ and 4-nitrobenzaldehyde $(10 \mathrm{mM})$. Green inverted triangles: addition of 100 mol\% aniline. Red circles: addition of $5 \mathrm{mM}$ Fmoc-aniline and $50 \mathrm{mM}$ DCv urea U1. Blue triangles: addition of $50 \mathrm{mM} \mathrm{DCv}$ urea U1. Violet squares: addition of $50 \mathrm{mM}$ bulky base 2.

Furthermore, we found the same phenomenon as the previously defined 'activation time' in the triggered systems where the acylhydrazone formation was tracked. For the system with the addition of the DCV hindered urea U1 (Figure 3, blue triangles), we observed an activation time of $101 \pm 25 \mathrm{~min}$, whereas for the system with the addition of DCv hindered urea U1 and Fmoc-aniline (Figure 3, red squares), we observed an activation time of $128 \pm 25 \mathrm{~min}$, which can be attributed to the delayed release of free bulky base $\mathbf{2}$ and aniline, respectively. For the anilinecatalysed reaction we observed a relatively short activation time of $48 \pm 25 \mathrm{~min}$, attributed to the initial reaction between aniline and 4-nitrobenzaldehyde to form the corresponding imine. Additionally, we decided to compare the maximum rates of the triggered and non-triggered systems to quantify the differences (Table 2). For the nontriggered systems, the highest conversion rate was achieved early on, whereas for the triggered systems it was achieved at the end of the activation time (see S.I.). It can be seen that the triggered aniline-release system shows a roughly two-fold increase in maximum rate of product formation compared to the traditional aniline-catalysed system, whereas it is roughly 12.5-times faster than the background reaction. These findings show that DCv ureas can not only be used for the triggered release of species protected with the Fmoc-group, but can also enhance traditional catalysis.

Table 2: maximum rates and activation times of formation of acylhydrazone from benzhydrazide $(10 \mathrm{mM})$ and 4-nitrobenzaldehyde (10 mM) in the presence of different catalytically active species as well as without any additive (background reaction) in $4 / 1$ DMSO- $\mathrm{d}_{6} / \mathrm{D}_{2} \mathrm{O}$, derived from the time derivative of the conversion graphs in Figure 3. For systems where no initial increase in reaction rate is observed, an activation time is not applicable (N/A).

\begin{tabular}{lll}
\hline System & Max. rate (mM/min) & $\begin{array}{l}\text { Activation } \\
\text { time (min) }\end{array}$ \\
\hline DCv hindered urea & $0.033 \pm 0.001$ & $128 \pm 25$ \\
U1 + Fmoc aniline & & \\
Bulky base 2 & $0.036 \pm 0.001$ & $\mathrm{~N} / \mathrm{A}$ \\
Aniline & $0.017 \pm 0.003$ & $48 \pm 25$ \\
DCv hindered urea & $0.0116 \pm 0.0003$ & $101 \pm 25$ \\
U1 & & \\
background & $0.00264 \pm 0.00003$ & $\mathrm{~N} / \mathrm{A}$ \\
\hline
\end{tabular}




\section{Triggered elimination for the release of nitrile- $\mathrm{N}$-oxides}

Next, we use an example in soft material formation to demonstrate the broad application area of the latent base present in the equilibria of DCv ureas. Here, the base eliminates hydrogen chloride from bis-chlorooximes to release nitrile- $N$-oxides in situ. These highly reactive nitrile- $N$-oxides can react with thiols to form thiohydroximates, which we apply as a crosslinking reaction in the formation of a polymer gel. To demonstrate this concept, we probed the formation of thiohydroximates via ${ }^{1} \mathrm{H}-\mathrm{NMR}$ spectroscopy in a small molecule test. The result can be seen in Figure 4. The formation of the bisthiohydroximate was confirmed via LCMS (calc. for $[\mathrm{M}+\mathrm{H}]^{+}:$: 429.12, observed: 429.10), however a clear designation of product signals in the ${ }^{1} \mathrm{H}-\mathrm{NMR}$ spectra is not possible due to interfering signals and side reactions. Side products that could be confirmed via LCMS are $N, N^{\prime}$-dibenzylurea (calc. for $[\mathrm{M}+\mathrm{H}]^{+}: 241.13$, observed: 241.14) and the thiol-isocyanate adduct (calc. for $[\mathrm{M}+\mathrm{H}]^{+}: 268.10$, observed: 267.96).

A)

A)

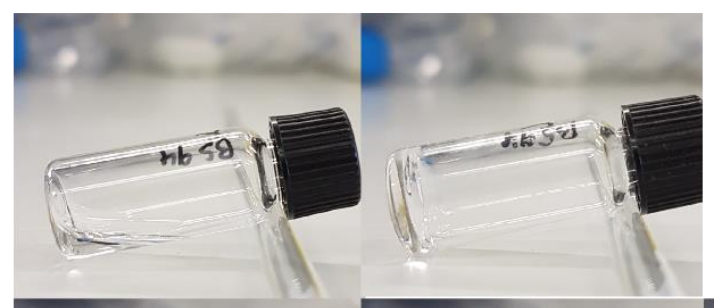

(1)

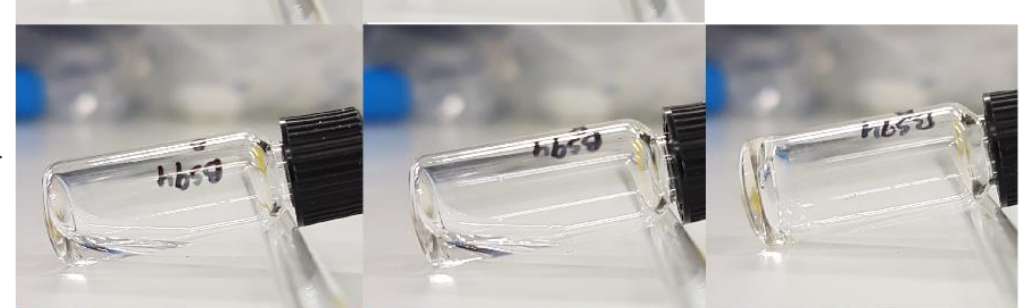

1.
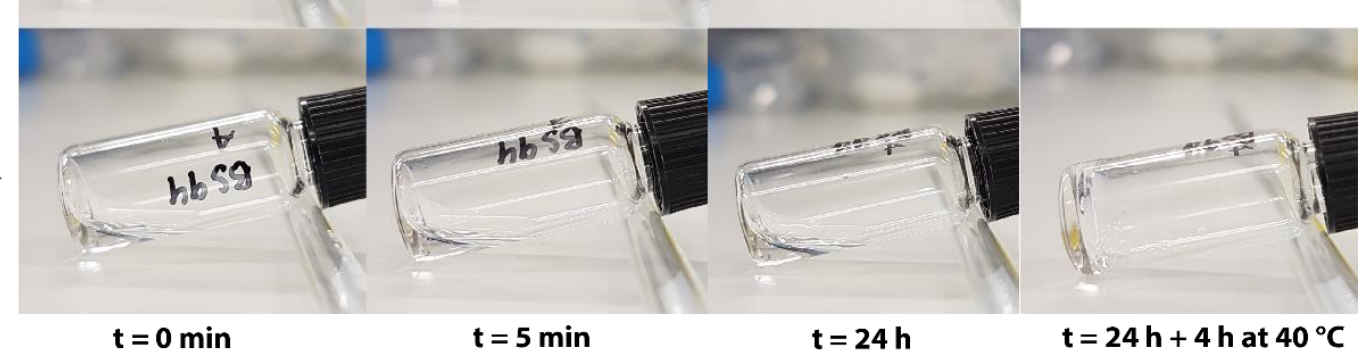

B)

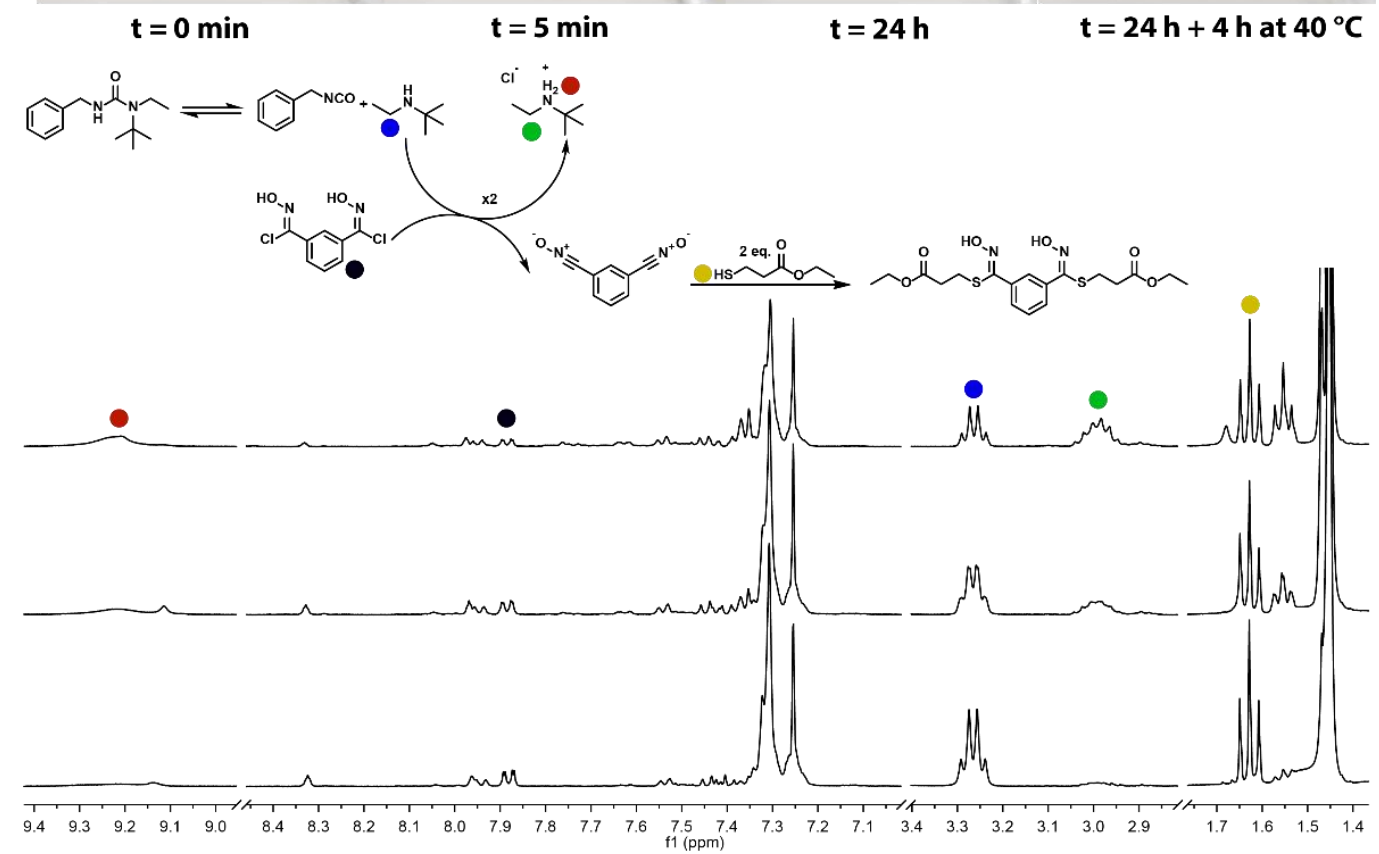

Figure 4: temperature-dependent gelation of a $10 \mathrm{wt} \%$ solution of bis-chlorooxime (2.0 eq., 1.0 eq. relative to thiol groups) and 4 -arm PEG thiol (1.0 eq.) in chloroform with the addition of 5 eq. (1.25 eq. relative to thiol and chlorooxime groups) of DCv ureas with different substituents. A) time- and temperaturedependent gelation upon addition of a free base and DCV ureas $\mathbf{U} \mathbf{1}$ and $\mathbf{U} 2$. B) reaction with a small molecule thiol tracked via ${ }^{1} \mathrm{H}-\mathrm{NMR}$. 
To put the concept of the in situ formation of nitrile- $N$-oxides to use, we decided to test the heat-triggered gelation of the generated bifunctional nitrile- $N$-oxides with tetrafunctional 4-arm PEG thiols $\left(\mathrm{M}_{\mathrm{w}}=10 \mathrm{~kg} / \mathrm{mol}\right)$. We hypothesised that the side reactions detected in the small molecule test should not cause issues as gelation with a 4-arm star polymer will occur even at low conversions. The starting materials were dissolved in chloroform to yield a 10 wt\% solution. Tests were performed with the ethyl-based $\mathbf{U} \mathbf{1}$ and iso-propyl-based U2 DCv ureas. The solutions were kept at room temperature overnight and heated to $40{ }^{\circ} \mathrm{C}$ thereafter.

Figure 4 shows that the ethyl-based DCv urea $\mathbf{U} 1$ is a good candidate to enable a temperature-triggered gelation via thiohydroximate formation. It can be seen that both experiments start with a clear solution of bischlorooxime, DCV urea and 4-arm PEG thiol. In the case of the iso-propyl based DCv urea U2, a colourless transparent gel is formed overnight at room temperature, where in the experiment with the ethyl-based DCv urea, the solution does not gel under the same conditions. Upon heating the latter sample to $40^{\circ} \mathrm{C}$ for 4 hours, a transparent gel ultimately forms as well. These findings can be attributed to the different equilibrium constants of the DCV ureas. For DCv urea U2, where the equilibrium is more on the side of the free base than for DCv urea U1, gelation can happen faster. We performed a control by adding identical equivalents of triethylamine as a free base to the solution. In this case, we observed gelation within 5 minutes under ambient conditions to form a transparent gel, suggesting that the gelation times can be tuned over a wide range, depending on whether a free base or DCv ureas with varying bulkiness are added to the solution of bischlorooxime and 4-arm PEG thiol.

\section{Conclusions}

We demonstrated that the species present in the equilibrium of DCV ureas can be used in reaction cascades and as organocatalysts on demand. We show that the base released in the urea equilibrium can be applied to 1 ) catalytically cleave the base-labile Fmoc protecting group, releasing active organocatalysts which in turn catalyse the formation of acylhydrazones above levels found for the free catalyst alone and 2) form highly reactive nitrile$\mathrm{N}$-oxides in situ which can lead to the gelation with thiols present in solution, forming a thiohydroximate polymer gel.

Further, we demonstrated that the temperature- and bulk-dependence of the urea equilibrium as well as the reactivity of the released isocyanate species towards nucleophiles can be employed to tune the urea equilibrium, reversibly and irreversibly, in a way that allows great control over the release of aniline and nitrile- $N$-oxides on demand.

We envisage that these findings have great potential in the of design responsive materials which possess useful functionalities on demand. Especially the sensitivity of the urea equilibrium at or near body temperature sets these triggered release-systems up for potential uses in biomedical materials.

Our group is currently working on applying these equilibria in crosslinked materials to design triggered, self-healing networks.

\section{Conflicts of interest}

There are no conflicts to declare.

\section{Acknowledgements}

This work has received funding from the European Union's Horizon 2020 research and innovation programme under the Marie Skłodowska-Curie grant agreement No 812868. We thank Dr. Stephen Eustace for help with the NMR measurements. 


\section{Graphical Abstract}

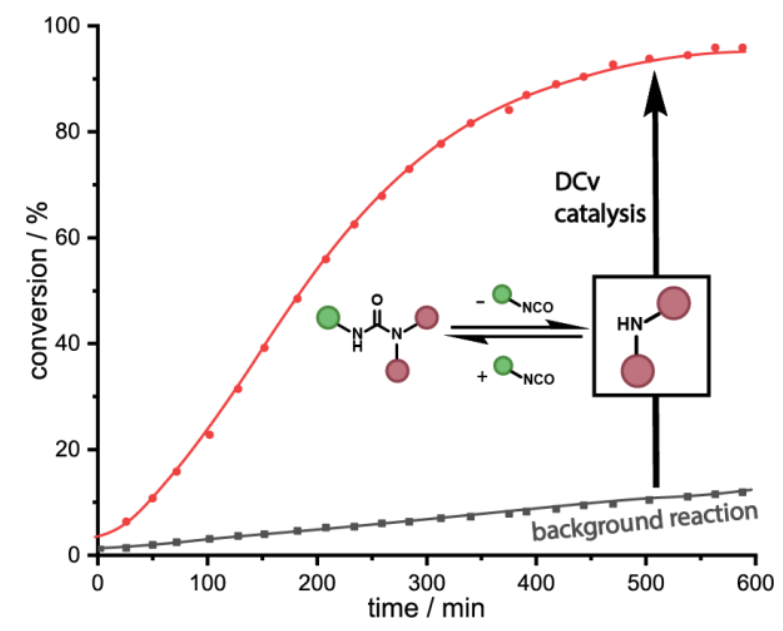

\section{Notes and references}

1 H. Ying, Y. Zhang and J. Cheng, Nat. Commun., 2014, 5, 1-7.

2 Y. Jin, C. Yu, R. J. Denman and W. Zhang, Chem. Soc. Rev., 2013, 42, 6634-6654.

3 J. M. Winne, L. Leibler and F. E. Du Prez, Polym. Chem., 2019, 10, 6091-6108.

4 P. G. M. Wuts and T. M. Greene, Greene's Protective Groups in Organic Synthesis, 2006, vol. 4.

5 M. Hutchby, C. E. Houlden, M. F. Haddow, S. N. G. Tyler, G. C. Lloyd-Jones and K. I. Booker-Milburn, Angew. Chemie - Int. Ed., 2012, 51, 548-551.

6 T. Shanmugam and A. S. Nasar, Macromol. Chem. Phys., 2008, 209, 651-665.

7 R. Spindler and J. M. J. Fréchet, Macromolecules, 1993, 26, 4809-4813.

8 K. A. Günay, P. Theato and H. A. Klok, J. Polym. Sci. Part A Polym. Chem., 2013, 51, 1-28.

9 M. S. Rolph, A. L. J. Markowska, C. N. Warriner and R. K. O’Reilly, Polym. Chem., 2016, 7, 7351-7364.

10 R. T. M. Jakobs, S. Ma and R. P. Sijbesma, ACS Macro Lett., 2013, 2, 613-616.

D. J. Adams, M. F. Butler, W. J. Frith, M. Kirkland, L. Mullen and P. Sanderson, Soft Matter, 2009, 5, 1856-1862.

L. A. Carpino and G. Y. Han, J. Org. Chem., 1972, 37, 3404-3409.

P. M. Pihko, I. Majander and A. Erkkilä, Top. Curr. Chem., 2009, 291, 29-75.

A. Erkkilä, I. Majander and P. M. Pihko, Chem. Rev., 2007, 107, 5416-5470.

Y. Zhou, I. Piergentili, J. Hong, M. P. van der Helm, M. Macchione, Y. Li, R. Eelkema and S. Luo, Org. Lett., 2020, 22, 6035-6040.

F. Trausel, C. Maity, J. M. Poolman, D. S. J. Kouwenberg, F. Versluis, J. H. Van Esch and R. Eelkema, Nat. Commun., 2017, 8, 1-7.

M. P. van der Helm, B. Klemm and R. Eelkema, Nat. Rev. Chem., 2019, 3, 491-508.

D. K. Kölmel and E. T. Kool, Chem. Rev., 2017, 117, 10358-10376.

D. Cerniauskaite, J. Rousseau, A. Sackus, P. Rollin and A. Tatibouët, European J. Org. Chem., 2011, 2293-2300.

R. Schaefer, M. R. Monaco, M. Li, A. Tirla, P. Rivera-Fuentes and H. Wennemers, J. Am. Chem. Soc., 2019, 141, 18644-18648.

T. Matsumura, Y. Koyama, S. Uchida, M. Yonekawa, T. Yui, O. Ishitani and T. Takata, Polym. J., 2014, 46, 609-616. M. H. Benn, Can. J. Chem., 1964, 42, 2393-2397.

Y. G. Lee, Y. Koyama, M. Yonekawa and T. Takata, Macromolecules, 2009, 42, 7709-7717.

H. Ying and J. Cheng, J. Am. Chem. Soc., 2014, 136, 16974-16977.

C. Maity, F. Trausel and R. Eelkema, Chem. Sci., 2018, 9, 5999-6005.

W. Xi, M. Krieger, C. J. Kloxin and C. N. Bowman, Chem. Commun., 2013, 49, 4504-4506.

G. Li, F. Trausel, M. van der Helm, B. Klemm, T. Breve, S. van Rossum, M. Hartono, H. Gerlings, M. Lovrak, J. van Esch and R. Eelkema, Angew. Chemie Int. Ed., 2021, 60, 2-10. 